\title{
Abordaje clínico del dolor lumbar crónico: síntesis de recomendaciones basadas en la evidencia de las guías de práctica clínica existentes
}

\author{
Clinical approach to chronic lumbar pain: a systematic review \\ of recommendations included in existing practice guidelines
}

\author{
D. Ángel García ${ }^{1}$, I. Martínez Nicolás ${ }^{1}$, P.J. Saturno Hernández ${ }^{1}$, F. López Soriano²
}

\section{RESUMEN}

Se realizó una revisión sistemática de guías de práctica clínica basadas en evidencia que tuvieran recomendaciones explícitas sobre el tratamiento del dolor lumbar crónico, con el objetivo de analizar su contenido y proveer una síntesis que sirviese para su traslación a la práctica. Las recomendaciones se analizaron y clasificaron según el tipo de tratamiento; se identificó el nivel de evidencia y fuerza de recomendación de las mismas, traduciéndolos a un sistema de gradación común. Ocho guías cumplieron los criterios de inclusión. El ejercicio y la escuela de espalda se perfilan como la terapia más efectiva. La farmacoterapia está indicada durante cortos periodos. Las guías analizadas ofrecen vías de acción similares frente a los principales tratamientos. Se han resumido las recomendaciones de forma clara y estructurada para permitir una elección clínica de los tratamientos más adecuados, evitar gastos y recursos en tratamientos inútiles y una recuperación del paciente más eficiente.

Palabras clave. Dolor lumbar crónico. Manejo del dolor. Guías de práctica clínica. Práctica basada en la evidencia.

\begin{abstract}
This paper presents a systematic review of clinical practice guidelines based on evidence, with explicit recommendations on the treatment of chronic low back pain. The main objective was to analyze their content and provide a synthesis in order to improve the translation of this evidence into practice. The recommendations were analyzed and then classified by treatment; evidence level and strength of recommendation were identified and translated into our gradation system. Eight guidelines met the inclusion criteria. Exercise and back school treatment are shown as the best therapies. Pharmacotherapy is indicated during short periods of time. The guidelines analyzed show similar therapeutic approaches. The recommendations have been synthesized in order to allow clinical selection of the best treatment and avoid bad practices with their corresponding costs, providing a more efficient management of the patient.
\end{abstract}

Keywords. Low back chronic pain. Pain management. Practice guideline. Evidence-based practice.
1. Departamento de Salud Pública y Medicina Preventiva. Universidad de Murcia.

2. Unidad del Dolor. Hospital Comarcal de Noroeste. Murcia.

Recepción: 14 de octubre de 2014

Aceptación provisional: 23 de diciembre de 2014

Aceptación definitiva: 9 de enero de 2015

\section{Correspondencia:}

Daniel Ángel García

Facultad de Medicina Universidad de Murcia

Departamento de Salud Pública y Medicina

Preventiva

Campus de Espinardo, s/n

30100 Murcia. España

E-mail: daniel.angel@um.es 


\section{INTRODUCCIÓN}

El dolor lumbar habitualmente se define como dolor, tensión muscular o rigidez localizado entre los márgenes costales y los pliegues glúteos, con o sin dolor (irradiado) en la pierna; y se clasifica como crónico cuando persiste más allá de 12 semanas ${ }^{1}$. Afecta a ambos géneros y a casi todos los rangos de edad, y es una importante causa de pérdida de calidad de vida debido al dolor y la limitación funcional que produce. Además genera importantes gastos directos (gastos de cuidados) e indirectos (absentismo laboral, etc.) ${ }^{2,3}$. La mayoría de los episodios agudos de lumbalgia se resuelven en un plazo menor de 2 semanas, pero la recurrencia el primer año es alta $(30-60 \%)^{4-6} ; \mathrm{y}$ en un tercio de los pacientes el episodio inicial de dolor persiste en el tiempo, cronificándose ${ }^{7}$. Afortunadamente, solo una pequeña parte de los pacientes sufren una discapacidad severa ${ }^{8}$.

Actualmente se estima que entre un cuarto y un tercio del gasto total de sanidad corresponde a este problema de salud ${ }^{9}$. Los gastos de atención y por discapacidad siguen aumentando con los años ${ }^{10}$. Los gastos indirectos, aun siendo difíciles de evaluar, se estima que superan el coste sanitario que supone esta enfermedad, debido principalmente a los días de trabajo perdidos ${ }^{3}$. Estos gastos son mayores a los de otras enfermedades equiparables en sintomatología y gravedad ${ }^{10,11}$, lo que pone sobre la pista de una pobre gestión de la enfermedad a varios niveles. La forma de abordar el manejo del dolor lumbar crónico (DLC) debe partir de la medicina basada en la evidencia. Transferir los hallazgos de la evidencia científica a la práctica clínica no es una tarea fácil, pero nos aporta ventajas importantes respecto a la práctica libre: un uso adecuado de los recursos, certeza del uso de terapias que dan resultado, y ante todo evitar vías de acción equivocadas o sin fundamento, que suponen un aumento del coste tanto económico como humano para el sistema sanitario y el paciente, respectivamente ${ }^{12,13}$.

Una de las principales herramientas que promueven la práctica basada en la evidencia son las guías de práctica clínica
(GPC). La calidad metodológica de éstas ha aumentado en los últimos años ${ }^{14,15}$ lo que permite basar las decisiones terapéuticas en evidencia sólida y relevante, pero el rápido aumento de GPC también se ha traducido en una importante variabilidad de los métodos de su desarrollo y, por lo tanto, en una variabilidad también de las recomendaciones clínicas por la ausencia de un desarrollo estandarizado ${ }^{16}$. Esta revisión tiene como objetivo identificar y analizar las guías de práctica clínica más recientes, para exponer de forma resumida y clara las recomendaciones que se pueden extraer para el tratamiento más adecuado del DLC.

\section{MATERIAL Y MÉTODO}

Se realizó una búsqueda sistemática en las bases de datos de Pubmed, Cochrane y ScienceDirect, así como en las siguientes bases de guías de práctica clínica y evidencia clínica: BMJ Evidence, SIGN, NZGG, NICE, AHRQ y Guiasalud. Se utilizaron los siguientes términos: "chronic low back pain", "chronic pain" (MeSH), "low back pain", "guideline", y "clinical practice guideline". Además se realizó una búsqueda manual de la bibliografía de los artículos más relevantes y en Google Scholar. La búsqueda se limitó en todas las bases de datos desde enero de 2003 a junio de 2013.

Se incluyeron las GPC que cumplieran los siguientes requisitos: a) estar dirigidas al tratamiento del dolor lumbar crónico, b) estar basadas en evidencia científica (ensayos clínicos, revisiones sistemáticas, metaanálisis), c) incluir niveles de evidencia y/o fuerza de recomendación, además de referencias claras, d) estar escritas en inglés o castellano.

Se evaluó la última edición de las guías disponibles y se tuvo en cuenta la información complementaria de las guías como los materiales de apoyo clínicos o resúmenes de recomendaciones. Adicionalmente, se realizó una evaluación crítica de la calidad metodológica de las GPC incluidas con la herramienta AGREE II en su versión en español ${ }^{17}$ por dos evaluadores independientes con experiencia previa en el uso de la 
herramienta. Se decidió no excluir ninguna guía tras la obtención de sus resultados.

Una vez identificadas las guías válidas, se analizaron las recomendaciones propuestas por las mismas, agrupándolas según el tipo de tratamiento: manejo general, fisioterapia, farmacoterapia, terapias alternativas, psicología y procedimientos invasivos. Se extrajeron solo las recomendaciones explícitas, considerando recomendación válida: la afirmación o enunciado fácilmente identificable que sintetice la evidencia de las terapias de las cuales debe hacerse uso, puede hacerse uso o se aconseja sobre su uso, ya sea de forma positiva o negativa. Se identificó el nivel de evidencia y fuerza de recomendación de cada una, y se tradujo a nuestro propio sistema unificado de gradación de la evidencia y fuerza de recomendación, desarrollado en base a la armonización de las propuestas de la U.S. Preventive Services Task Force ${ }^{18}$, y los sistemas GRADE ${ }^{19}$, y $\mathrm{SIGN}^{20}$. El análisis de la evidencia se ha realizado desde el punto de vista del usuario de la GPC, traduciendo el nivel de evidencia y fuerza de recomendación directamente a partir de los mostrados por cada guía.

Este proceso permite comparar las recomendaciones entre guías en cuanto al nivel de evidencia y fuerza de recomendación y analizar la variabilidad que se produce a la hora de emitir las recomendaciones. A partir de la comparación de la información en las distintas guías sobre cada tratamiento, se elaboraron las recomendaciones finales para el correcto manejo del DLC. Para el establecimiento del NE y FR de estas recomendaciones (Tabla 1) se tuvo en cuenta si la evidencia que soportaba las recomendaciones era constante en todas las guías y la variación de ésta a lo largo del tiempo.

\section{RESULTADOS}

Se obtuvieron 746 resultados en la búsqueda de los cuales se identificaron 20 potenciales guías, de las que fueron excluidas 12. Finalmente 8 fueron incluidas ${ }^{21-29}$ (Fig. 1). La calidad metodológica de estas GPC es muy diversa, siendo más marcadas las diferencias entre guías en los dominios de "Aplicabilidad" (puntuación media del dominio de 39,32\% y desviación estándar de 25,48\%) e "Independencia Editorial" (media de 51,56\%; desviación estándar de $32,40 \%)$. La puntuación media de todas las guías para cada uno de los demás dominios "Alcance y Objetivo; Participación de los Implicados; Rigor en la Elaboración; y Claridad de Presentación" es igual o mayor de 51,39\% (con una desviación estándar menor o igual a $19,79 \%$ en cada uno). Se presenta un análisis global de cada guía en base al número de dominios que superan una puntuación del $50 \%$ en la figura 2 según los resultados del AGREE II.

\section{Variabilidad en las guías de práctica clínica}

La tabla 2 muestra los resultados con los niveles de evidencia y fuerza de recomendación para cada una de las terapias en las distintas guías. Solo tres terapias han sido incluidas en todas las guías: el ejercicio, las manipulaciones y la tracción. La primera muestra alto nivel de evidencia en todas las guías, y está indicada como pilar fundamental del tratamiento. La manipulación está indicada en poblaciones específicas, pero existe controversia para su recomendación. En cambio se recomienda no usar la tracción en el DLC por no resultar efectiva e incurrir en posibles afectos adversos ${ }^{22,24}$.

Además de los mencionados, encontramos más tipos de abordaje en todas las guías si obviamos la de APTA $2012^{29}$ (orientada específicamente a tratamiento basado en terapia manual): masaje, sobre el que no hay acuerdo entre las guías, y TENS (Transcutaneous Electrical Nerve Stimulation), que no se recomienda su uso por no mostrar beneficios.

Cinco terapias han mostrado resultados consistentes para recomendar su uso y aparecen en al menos 5 guías: ejercicio, abordaje multidisciplinar, escuela de espalda, opioides y antidepresivos. Diecinueve de los 46 tipos de terapias identificadas en las guías presentan resultados consistentes en los niveles de evidencia y fuerza de recomendación, 14 recomendaciones a favor, 3 en contra y 4 que no se pueden 
Tabla 1. Síntesis de recomendaciones para el tratamiento del dolor lumbar crónico

\section{Recomendaciones con fuerza A (Debe hacerse) constante en las guías}

\begin{tabular}{|c|c|}
\hline 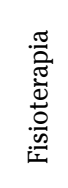 & $\begin{array}{l}\text { Los pacientes deben iniciar un programa de ejercicio. Es aconsejable establecer una rutina pro- } \\
\text { gresiva para evitar exacerbar el dolor. Se obtienen mejores resultados con rutinas estructuradas } \\
\text { y supervisión por parte de especialistas. Se debe vigilar que el ejercicio no aumente el dolor del } \\
\text { paciente. Ningún ejercicio ha demostrado ser mejor que otro; se han encontrado resultados positi- } \\
\text { vos con ejercicios de fuerza, resistencia, coordinación, de flexión, de extensión (McKenzie), isoci- } \\
\text { néticos y trabajo aeróbico de bajo impacto en la zona lumbar dependiendo del perfil del paciente. }\end{array}$ \\
\hline
\end{tabular}

\section{Recomendaciones con fuerza B (puede hacerse) constante en las guías}

El tratamiento multidisciplinar es necesario debido a los múltiples mecanismos de acción del dolor crónico. Las terapias combinadas también mejoran los resultados.

La Escuela de espalda es igual de eficaz que otros tratamientos conservadores, combina educación del paciente en el dolor lumbar, ejercicios que puede realizar el paciente e higiene postural. Depende del enfoque se pueden incluir más terapias (ejercicio aeróbico, tratamiento cognitivocomportamental...).

La educación del paciente sobre su enfermedad y autocuidado debe ser parte de la terapia, ya sea como parte de programas como el de escuela de espalda, o como una intervención más.

Se debe recomendar a los pacientes que mantengan una vida activa. $\quad$ A2

Ejercicios de la modalidad McKenzie están indicados en el tratamiento del dolor lumbar crónico. A2

: :

- Menores de 40 años.

- Test de inestabilidad en prono positivo.

- Presencia de movimientos aberrantes durante la evaluación del movimiento.

- "Straight leg raise" mayor de $91^{\circ}$

Los Antidepresivos tricíclicos tienen un efecto pequeño-moderado en el alivio del dolor lumbar crónico, con dosis más pequeñas que las usadas para la depresión. Son más efectivos en el tratamiento de DLC que curse con depresión y/o trastornos del sueño.

Titular desde 10 a 25 miligramos (por la noche), subiendo $25 \mathrm{mg}$ a la semana hasta dosis terapéutica o $100 \mathrm{mg}$ (amitriptilina, nortriptilina).

Relajantes musculares: La ciclobenzaprina puede ser apropiada en pacientes seleccionados que muestren cuadro de espasmo muscular. No está recomendado su uso crónico. Controlar posible abuso. De 10 a $30 \mathrm{mg}$ al día. Los mayores beneficios se han visto dentro de la primera semana. No utilizar más de 2 semanas.

El Paracetamol puede ser una primera opción (antes que AINEs) para el control del dolor durante periodos cortos. Hasta $1000 \mathrm{mg}$ tres veces al día (máximo $3000 \mathrm{mg}$ al día).

Los Opioides suaves deben ser utilizados solo cuando otras opciones terapéuticas hayan fracasado. Durante periodos muy cortos de tiempo (1-2 semanas). Evitar este tratamiento en pacientes con antecedentes de abuso de alguna sustancia o problemas psiquiátricos. Debido a la complejidad del tratamiento del dolor crónico con opioides se aconseja la consulta de la guía Canadian Guideline for Safe and Effective Use of Opioids for Chronic Non-Cancer Pain, disponible en: http:// nationalpaincentre.mcmaster.ca/opioid/

Las variantes Viniyoga e lyengar del Yoga pueden ayudar al tratamiento de la lumbalgia crónica.

Las siguientes medicinas herbales se pueden usar en brotes agudos de la lumbalgia crónica*:

- Extracto acuoso de Harpagophytum procumbens (Devil's Claw, grappleplant, wood spider) a dosis estandarizada diaria de $50 \mathrm{mg}$ harpagoside.

- Una combinación de extracto de Salixdaphnoides y Salix purpurea (sauce rojo, sauce púrpura) a dosis estandarizada diaria de $240 \mathrm{mg}$ salicina/día.

- Un emplasto de Capsicum frutescens (pimiento picante, chile rojo, pimiento de tabasco).

El uso de la capsicina debería considerarse en pacientes con dolor moderado a severo que no han respondido a otros tratamientos.

$\vec{\omega} \quad$ La inclusión del paciente en un tratamiento cognitivo-comportamental (grupal o individual) dirigido por un especialista está recomendada, como parte de un conjunto de terapias. 
Recomendaciones con fuerza B (puede hacerse) no constante en las guías.

즐 Referir a cirugía a pacientes con dolor severo (EVA $\geq 7)$ y ha completado un set de tratamiento que incluya terapia física y psicológica sin éxito en el alivio del dolor.

Los pacientes que no responden al tratamiento pueden beneficiarse de la manipulación por parte de un profesional cualificado. La manipulación está contraindicada en pacientes con signos neu- A2 rológicos.

El masaje terapéutico está indicado como coadyuvante, pero en monoterapia no es mejor que otros tratamientos.

\begin{tabular}{|c|c|}
\hline $\begin{array}{l}\stackrel{\dot{0}}{0} \\
\stackrel{0}{0} \\
\stackrel{\Xi}{0}\end{array}$ & $\begin{array}{l}\text { Los AINEs pueden ser usados durante periodos cortos para reducir el dolor. Nunca más de tres } \\
\text { meses. Ningún AINE es superior a otro. Un inhibidor de la bomba de protones debe considerarse } \\
\text { como coadyuvante en pacientes mayores de } 45 \text { años. } \\
\text { - Ibuprofeno } 800 \mathrm{mg} \text { tres veces al día (máximo } 800 \mathrm{mg} 4 \text { veces al día). } \\
\text { - Dicoflenaco hasta } 50 \mathrm{mg} \text { tres veces al día. }\end{array}$ \\
\hline
\end{tabular}

$\varangle \quad$ La acupuntura está indicada como parte de la terapia para el dolor crónico lumbar.

\section{Recomendaciones con fuerza C (no hay evidencia suficiente o existe evidencia contradictoria)}

No se puede recomendar el uso de TENS, Láser de Baja intensidad, Ultrasonidos, Neurorreflexoterapia, PENS, Biofeedback, calor, uso de colchones firmes, soportes lumbares, corrientes interferenciales, onda corta, plantillas, relajantes musculares, proloterapia, IRSS, IRSN, glucocorticoides intradiscales, corticoides intrarticulares en articulaciones facetarias, infiltración epidural de corticoesteriodes, denervación por radiofrecuencia, infiltración de puntos gatillo, gabapentina, vitamina Be infiltraciones de oxígeno y ozono.

\begin{tabular}{lc}
\hline \multicolumn{2}{c}{ Recomendaciones con fuerza D (No debe hacerse) } \\
\hline No se debe recomendar el reposo (total) en cama. & A2 \\
\hline No se debe realizar tracción como terapia para el dolor lumbar crónico. & A2
\end{tabular}

*Esta recomendación sólo aparece en una guía, por lo que su NE/FR no cambia pero tampoco se puede comparar. Abreviaturas. NE: Nivel de Evidencia; Gral.: Manejo General; Psi.: Piscología; Farmacotp.: farmacoterapia; TA.: Terapias Alternativas; AINE: Antiinflamatorio no esteroideo; DLC: Dolor Crónico Lumbar EVA: Escala Visual Analógica; TENS: Neuroestimulación eléctrica transcutanea; IRSS: Inhibidores de la receptación de la serotonina; IRSN: Inhibidores de la receptación de la noradrenalina;PENS: Neuroestimulación eléctrica percutánea.

recomendar por falta de evidencia. Todas las demás terapias muestran diferentes niveles de evidencia e incluso fuerza de recomendación.

\section{Evidencia en el abordaje clínico del dolor lumbar crónico}

En la tabla 1 se muestra la síntesis de las recomendaciones finales elaboradas para cada tipo de tratamiento. Se puede ver que el abordaje general del DLC debe realizarse de forma multidisciplinar debido a los múltiples mecanismos de acción del dolor crónico. Es necesario proveer medidas educativas al paciente y consejos que promuevan la vida activa. La indicación para referir al paciente al especialista y evaluar la posibilidad de someterlo a cirugía debe hacerse en caso de fallo de las demás terapias en el control del dolor y si el paciente presenta dolor severo (EVA $\geq 7$ ). No se debe recomendar el reposo en cama, y no hay evidencia suficiente para la recomendación del uso de colchones firmes y plantillas.

\section{Tratamiento no farmacológico}

El ejercicio y la escuela de espalda muestran recomendaciones con alto nivel de evidencia y fuerza de recomendación constan- 
Tabla 2. Recomendaciones según su nivel de evidencia y fuerza de recomendación

\begin{tabular}{|c|c|c|c|c|c|c|c|c|c|c|c|c|c|c|c|c|c|}
\hline & & \multicolumn{2}{|c|}{$\begin{array}{c}\text { Holanda } \\
\mathbf{2 0 0 3}^{21}\end{array}$} & \multicolumn{2}{|c|}{$\begin{array}{c}\text { Europa } \\
2006^{22} \\
\end{array}$} & \multicolumn{2}{|c|}{$\begin{array}{l}\text { CLIP } \\
2007^{23}\end{array}$} & \multicolumn{2}{|c|}{$\begin{array}{c}\text { ACP/APS } \\
\mathbf{2 0 0 7}^{24,25}\end{array}$} & \multicolumn{2}{|c|}{$\begin{array}{c}\text { Euskadi } \\
2007^{26}\end{array}$} & \multicolumn{2}{|c|}{$\begin{array}{l}\text { NICE } \\
2009^{27}\end{array}$} & \multicolumn{2}{|c|}{$\begin{array}{c}\text { Alberta } \\
2011^{28} \\
\end{array}$} & \multicolumn{2}{|c|}{$\begin{array}{l}\text { APTA } \\
2012^{29}\end{array}$} \\
\hline & & $\mathrm{NE}$ & FR & NE & FR & NE & FR & $\mathrm{NE}$ & FR & NE & FR & $\mathrm{NE}$ & FR & $\mathrm{NE}$ & FR & $\mathrm{NE}$ & FR \\
\hline \multirow{8}{*}{ 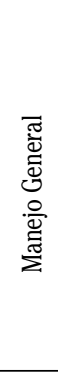 } & Colchones firmes & & & & & & & $\mathrm{C} 2$ & $\mathrm{D}$ & & & & & & & & \\
\hline & Educación & & & $\mathrm{A} 2$ & $\mathrm{~B}$ & & & $\mathrm{~A} 2$ & B & & & & & $\mathrm{A} 2$ & B & $\mathrm{A} 2$ & B \\
\hline & Fajas lumbares (soportes) & & & & & $\mathrm{C} 2$ & I & $\mathrm{C} 2$ & $\mathrm{C}$ & $\mathrm{C} 2$ & $\mathrm{D}$ & $\mathrm{C} 2$ & $\mathrm{C}$ & $\mathrm{A} 2$ & $\mathrm{D}$ & & \\
\hline & Mantener vida activa & $\mathrm{A} 2$ & B & $\mathrm{A} 2$ & $\mathrm{~B}$ & & & & & $\mathrm{~A} 2$ & B & & & & & & \\
\hline & Plantillas & & & & & & & & & & & & & $\mathrm{A} 3$ & $\mathrm{D}$ & & \\
\hline & $\begin{array}{l}\text { Referir a cirugía si tratamiento no } \\
\text { efectivo }\end{array}$ & & & & & & & & & $\mathrm{C} 2$ & $\mathrm{D}$ & $\mathrm{A} 2$ & B & $\mathrm{A} 2$ & B & & \\
\hline & Reposo en cama & & & & & $\mathrm{A} 2$ & $\mathrm{D}$ & & & $\mathrm{A} 2$ & $\mathrm{D}$ & & & & & $\mathrm{A} 2$ & $\mathrm{D}$ \\
\hline & Tratamiento multidisciplinar & & & $\mathrm{A} 2$ & A & $\mathrm{A} 2$ & $\mathrm{~A}$ & $\mathrm{~A} 2$ & B & & & A3 & $\mathrm{A}$ & $\mathrm{A} 2$ & B & & \\
\hline \multirow{16}{*}{ 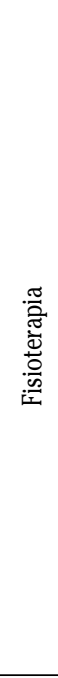 } & Abordaje de McKenzie & & & & & $\mathrm{A} 2$ & $\mathrm{~B}$ & & & & & & & & & $\mathrm{~A} 2$ & A \\
\hline & Biofeedback & $\mathrm{C} 2$ & $\mathrm{C}$ & & & & & $\mathrm{C} 2$ & $\mathrm{C}$ & & & & & & & & \\
\hline & Calor & & & $\mathrm{C} 2$ & I & & & & & $\mathrm{C} 2$ & $\mathrm{C}$ & & & & & & \\
\hline & Corrientes interferenciales & & & $\mathrm{D}$ & I & & & $\mathrm{C} 2$ & $\mathrm{C}$ & & & $\mathrm{D}$ & I & $\mathrm{D}$ & I & & \\
\hline & Ejercicio & $\mathrm{A} 2$ & A & $\mathrm{A} 2$ & $\mathrm{~A}$ & $\mathrm{~A} 2$ & $\mathrm{~A}$ & $\mathrm{~A} 2$ & B & $\mathrm{A} 2$ & $\mathrm{~A}$ & $\mathrm{Al}$ & B & $\mathrm{A} 2$ & B & $\mathrm{A} 1$ & A \\
\hline & Ejercicios estabilización & & & & & & & & & & & & & & & $\mathrm{A} 2$ & B \\
\hline & Escuela De espalda & & & $\mathrm{A} 2$ & $\mathrm{~B}$ & $\mathrm{~A} 2$ & $\mathrm{~B}$ & $\mathrm{~A} 2$ & $\mathrm{~B}$ & $\mathrm{~A} 2$ & B & $\mathrm{A} 2$ & $\mathrm{~B}$ & & & $\mathrm{~A} 2$ & B \\
\hline & Láser De baja intensidad & $\mathrm{C} 1$ & $\mathrm{C}$ & $\mathrm{C} 2$ & $\mathrm{C}$ & & & $\mathrm{C} 2$ & $\mathrm{C}$ & & & $\mathrm{A} 2$ & $\mathrm{D}$ & $\mathrm{C} 2$ & $\mathrm{C}$ & & \\
\hline & Manipulación & & & $\mathrm{A} 2$ & $\mathrm{~B}$ & $\mathrm{~A} 1$ & $\mathrm{~B}$ & $\mathrm{~A} 2$ & B & $\mathrm{A} 2$ & $\mathrm{D}$ & $\mathrm{A} 2$ & B & $\mathrm{C} 2$ & $\mathrm{C}$ & $\mathrm{A} 2$ & B \\
\hline & Masaje & $\mathrm{C} 2$ & $\mathrm{C}$ & $\mathrm{C} 2$ & $\mathrm{C}$ & $\mathrm{A} 2$ & $\mathrm{~B}$ & $\mathrm{~A} 2$ & $\mathrm{~B}$ & $\mathrm{C} 2$ & $\mathrm{C}$ & $\mathrm{A} 2$ & B & $\mathrm{A} 2$ & $\mathrm{~B}$ & & \\
\hline & Movilización & & & $\mathrm{A} 2$ & $\mathrm{~B}$ & & & & & & & & & $\mathrm{C} 2$ & $\mathrm{C}$ & & \\
\hline & Onda corta & & & $\mathrm{C} 2$ & I & & & $\mathrm{C} 2$ & $\mathrm{C}$ & & & & & & & & \\
\hline & PENS & & & $\mathrm{A} 2$ & $\mathrm{~B}$ & & & & & & & $\mathrm{C} 2$ & $\mathrm{C}$ & & & & \\
\hline & TENS & $\mathrm{C} 2$ & $\mathrm{C}$ & $\mathrm{A} 2$ & $\mathrm{D}$ & $\mathrm{A} 2$ & $\mathrm{D}$ & $\mathrm{C} 2$ & $\mathrm{C}$ & $\mathrm{C} 2$ & $\mathrm{D}$ & $\mathrm{C} 2$ & $\mathrm{C}$ & $\mathrm{A} 2$ & $\mathrm{D}$ & & \\
\hline & Tracción & $\mathrm{C} 2$ & $\mathrm{C}$ & $\mathrm{C} 2$ & $\mathrm{C}$ & $\mathrm{A} 2$ & $\mathrm{D}$ & $\mathrm{C} 2$ & $\mathrm{D}$ & $\mathrm{A} 2$ & $\mathrm{D}$ & $\mathrm{A} 2$ & $\mathrm{D}$ & $\mathrm{A} 2$ & $\mathrm{D}$ & $\mathrm{C} 2$ & $\mathrm{C}$ \\
\hline & Ultrasonidos & $\mathrm{C} 2$ & $\mathrm{C}$ & $\mathrm{C} 2$ & I & $\mathrm{A} 2$ & $\mathrm{D}$ & $\mathrm{C} 2$ & $\mathrm{C}$ & & & $\mathrm{D}$ & I & $\mathrm{C} 2$ & $\mathrm{C}$ & & \\
\hline \multirow{10}{*}{ 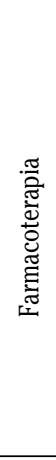 } & AINESs & & & $\mathrm{A} 2$ & $\mathrm{~A}$ & $\mathrm{~A} 2$ & $\mathrm{~B}$ & $\mathrm{~A} 2$ & $\mathrm{~B}$ & $\mathrm{C} 2$ & $\mathrm{C}$ & $\mathrm{A} 2$ & $\mathrm{~B}$ & $\mathrm{~A} 2$ & $\mathrm{~B}$ & & \\
\hline & Antidepresivos (IRSS/IRSN) & & & $\mathrm{A} 2$ & $\mathrm{~A}$ & $\mathrm{~A} 2$ & $\mathrm{~B}$ & & & $\mathrm{D}$ & $\mathrm{I}$ & $\mathrm{C} 2$ & $\mathrm{C}$ & $\mathrm{C} 2$ & $\mathrm{D}$ & & \\
\hline & $\begin{array}{l}\text { Antidepresivos tricíclicos y } \\
\text { tetracíclicos }\end{array}$ & & & & & $\mathrm{A} 2$ & B & $\mathrm{A} 2$ & B & $\mathrm{A} 2$ & B & $\mathrm{A} 2$ & B & $\mathrm{A} 2$ & B & & \\
\hline & Benzodiazepinas & & & $\mathrm{A} 2$ & $\mathrm{~B}$ & & & $\mathrm{~A} 2$ & B & $\mathrm{A} 2$ & B & & & $\mathrm{A} 2$ & B & & \\
\hline & Capsaicina & & & $\mathrm{A} 2$ & $\mathrm{~B}$ & & & & & $\mathrm{~A} 2$ & B & & & & & & \\
\hline & Gabapentina & & & $\mathrm{C} 2$ & $\mathrm{C}$ & & & $\mathrm{C} 2$ & $\mathrm{C}$ & & & & & & & & \\
\hline & Opioides suaves & & & $\mathrm{A} 2$ & $\mathrm{~B}$ & $\mathrm{~A} 2$ & $\mathrm{~B}$ & $\mathrm{~A} 2$ & $\mathrm{~B}$ & $\mathrm{~A} 2$ & $\mathrm{~B}$ & $\mathrm{~A} 2$ & $\mathrm{~B}$ & $\mathrm{~A} 2$ & $\mathrm{~B}$ & & \\
\hline & Paracetamol & & & & & & & $\mathrm{A} 2$ & $\mathrm{~B}$ & $\mathrm{~A} 2$ & $\mathrm{~B}$ & $\mathrm{~A} 2$ & $\mathrm{~A}$ & $\mathrm{~A} 2$ & $\mathrm{~B}$ & & \\
\hline & Relajantes musculares & & & $\mathrm{A} 2$ & $\mathrm{~B}$ & $\mathrm{~A} 2$ & B & $\mathrm{C} 2$ & $\mathrm{C}$ & & & & & & & & \\
\hline & Vitamina B & & & & & & & & & $\mathrm{C} 2$ & $\mathrm{D}$ & & & & & & \\
\hline \multirow{4}{*}{ 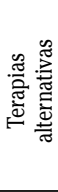 } & Acupuntura & & & $\mathrm{C} 2$ & $\mathrm{C}$ & $\mathrm{A} 2$ & B & $\mathrm{A} 2$ & B & $\mathrm{C} 2$ & $\mathrm{D}$ & $\mathrm{A} 1$ & A & $\mathrm{A} 2$ & B & & \\
\hline & Medicina natural & & & & & & & & & & & & & $\mathrm{A} 2$ & B & & \\
\hline & Neuroreflexoterapia & & & $\mathrm{A} 2$ & $\mathrm{~B}$ & $\mathrm{D}$ & $\mathrm{I}$ & & & $\mathrm{C} 2$ & I & $\mathrm{C} 2$ & $\mathrm{C}$ & & & & \\
\hline & Yoga & & & & & & & A3 & B & & & & & $\mathrm{A} 2$ & B & & \\
\hline Psic. & $\begin{array}{l}\text { Tratamiento cognitivo } \\
\text { comportamental }\end{array}$ & $\mathrm{A} 1$ & B & A2 & A & A2 & A & $\mathrm{A} 2$ & B & & & $\mathrm{C} 2$ & C & $\mathrm{A} 2$ & B & & \\
\hline
\end{tabular}




\begin{tabular}{|c|c|c|c|c|c|c|c|c|c|c|c|c|c|c|c|c|c|}
\hline & & \multicolumn{2}{|c|}{$\begin{array}{c}\text { Holanda } \\
2003^{21}\end{array}$} & \multicolumn{2}{|c|}{$\begin{array}{c}\text { Europa } \\
2006^{22}\end{array}$} & \multicolumn{2}{|c|}{$\begin{array}{c}\text { CLIP } \\
2007^{23}\end{array}$} & \multicolumn{2}{|c|}{$\begin{array}{c}\text { ACP/APS } \\
\mathbf{2 0 0 7}^{24,25}\end{array}$} & \multicolumn{2}{|c|}{$\begin{array}{c}\text { Euskadi } \\
\mathbf{2 0 0 7}^{26}\end{array}$} & \multicolumn{2}{|c|}{$\begin{array}{l}\text { NICE } \\
2009^{27}\end{array}$} & \multicolumn{2}{|c|}{$\begin{array}{c}\text { Alberta } \\
\text { 2011 }^{28}\end{array}$} & \multicolumn{2}{|c|}{$\begin{array}{l}\text { APTA } \\
\text { 2012 }^{29}\end{array}$} \\
\hline & & $\mathrm{NE}$ & FR & NE & FR & $\mathrm{NE}$ & FR & $\mathrm{NE}$ & FR & $\mathrm{NE}$ & FR & NE & FR & NE & FR & NE & FR \\
\hline \multirow{7}{*}{ 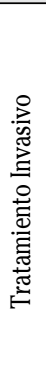 } & Denervación con radiofrecuencia & & & $\mathrm{C} 2$ & I & $\mathrm{A} 2$ & B & & & & & & & & & & \\
\hline & Infiltración de puntos gatillo & & & $\mathrm{C} 2$ & I & $\mathrm{A} 2$ & $\mathrm{~B}$ & & & $\mathrm{C} 2$ & $\mathrm{D}$ & & & & & & \\
\hline & $\begin{array}{l}\text { Infiltración epidural de } \\
\text { corticoesteroides }\end{array}$ & & & $\mathrm{D}$ & I & $\mathrm{A} 2$ & B & & & $\mathrm{C} 2$ & $\mathrm{D}$ & & & $\mathrm{A} 2$ & B & & \\
\hline & Infiltraciones oxigeno/ozono & & & & & & & & & B3 & $\mathrm{D}$ & & & & & & \\
\hline & $\begin{array}{l}\text { Inyección intradiscal de } \\
\text { glucocorticoides }\end{array}$ & & & $\mathrm{A} 2$ & $\mathrm{D}$ & $\mathrm{C} 2$ & $\mathrm{C}$ & & & $\mathrm{C} 2$ & $\mathrm{D}$ & $\mathrm{C} 2$ & $\mathrm{C}$ & & & & \\
\hline & $\begin{array}{l}\text { Inyecciones esclerosantes } \\
\text { (proloterapia) }\end{array}$ & & & $\mathrm{A} 2$ & $\mathrm{D}$ & $\mathrm{D}$ & I & & & $\mathrm{C} 2$ & $\mathrm{D}$ & $\mathrm{C} 2$ & $\mathrm{C}$ & $\mathrm{C} 2$ & $\mathrm{D}$ & & \\
\hline & Inyecciones IA de corticoides en $\mathrm{AF}$ & & & $\mathrm{D}$ & $\mathrm{I}$ & $\mathrm{A} 2$ & $\mathrm{D}$ & & & $\mathrm{C} 2$ & $\mathrm{D}$ & $\mathrm{C} 2$ & $\mathrm{D}$ & & & & \\
\hline
\end{tabular}

NE: Nivel de Evidencia; FR: Fuerza de recomendación; AINE: Antiinflamatorio no esteroideo; IA: Intra Articular; AF: Articulaciones Facetarias; IRSS: Inhibidores de la receptación de la serotonina; IRSN: Inhibidores de la receptación de la noradrenalina; tto: tratamiento;PENS: Neuroestimulación eléctrica percutánea.

tes en todas las GPC. Estos tratamientos, además de ser efectivos en el tratamiento del DLC, son seguros pues presentan un bajo nivel de efectos secundarios.

El yoga, la educación del paciente en caso de no tener acceso a la escuela de espalda, ejercicios de estabilización lumbar, abordaje de McKenzie y promover la vida activa son las terapias que a pesar de no estar reflejadas en todas las guías tienen un nivel de evidencia y fuerza de recomendación positiva constante. En cuanto a otros tratamientos no farmacológicos, encontramos que la terapia cognitivo-comportamental presenta recomendaciones a favor en todas las guías menos en una ${ }^{27}$; en esta guía se indica que los resultados de esta terapia utilizada de forma aislada no son significativos, por lo que deben ser usadas en combinación con otras terapias, en cuyo caso la recomendación de la guía es a favor de su uso.

Existe controversia en la acupuntura, el masaje, la manipulación y la movilización en las distintas guías, su éxito aparece ligado a si el grupo de pacientes a los que se aplica el tratamiento es el indicado, por lo que su uso generalizado no puede ser recomendado. Existe evidencia contradictoria o no relevante para láser de baja intensidad, neurorreflexoterapia, PENS, biofeedback, soportes lumbares, corrientes interferenciales, onda corta, TENS, ultrasonidos. En cualquier caso no se debe utilizar la tracción para tratar el DLC.

\section{Tratamiento farmacológico}

Atendiendo a los tratamientos farmacológicos, el paracetamol se muestra efectivo en el control del dolor, pero su uso está limitado a periodos cortos de tiempo. El uso de antiinflamatorios no esteroideos (AINE) se aconseja en todas las guías menos una que afirma que no hay suficiente evidencia de su eficacia ${ }^{26}$. Otra opción son los antidepresivos tricíclicos, como la amitriptilina o la nortriptilina, aunque en ambos casos su eficacia está solo confirmada durante periodos no superiores a tres meses. La última línea de acción son los opioides suaves, cuando el dolor no se puede controlar de otra manera. Las guías que los recomiendan de forma positiva hacen alusión a que los estudios disponibles solo aportan evidencia de intervenciones cortas, por lo que no están registrados los efectos a largo plazo, y por lo tanto solo deberían ser usados en caso de fracaso de las otras terapias, durante periodos cortos de tiempo y en pacientes seleccionados cuidadosamente.

\section{Otras opciones terapéuticas}

En cuanto a los remedios naturales: el extracto acuoso de Harpagophytum procumbens, la combinación de extracto de Salix daphnoides y Salix purpurea, y los emplastos de Capsicum frutescens tienen un efecto moderado sobre el alivio del dolor. 


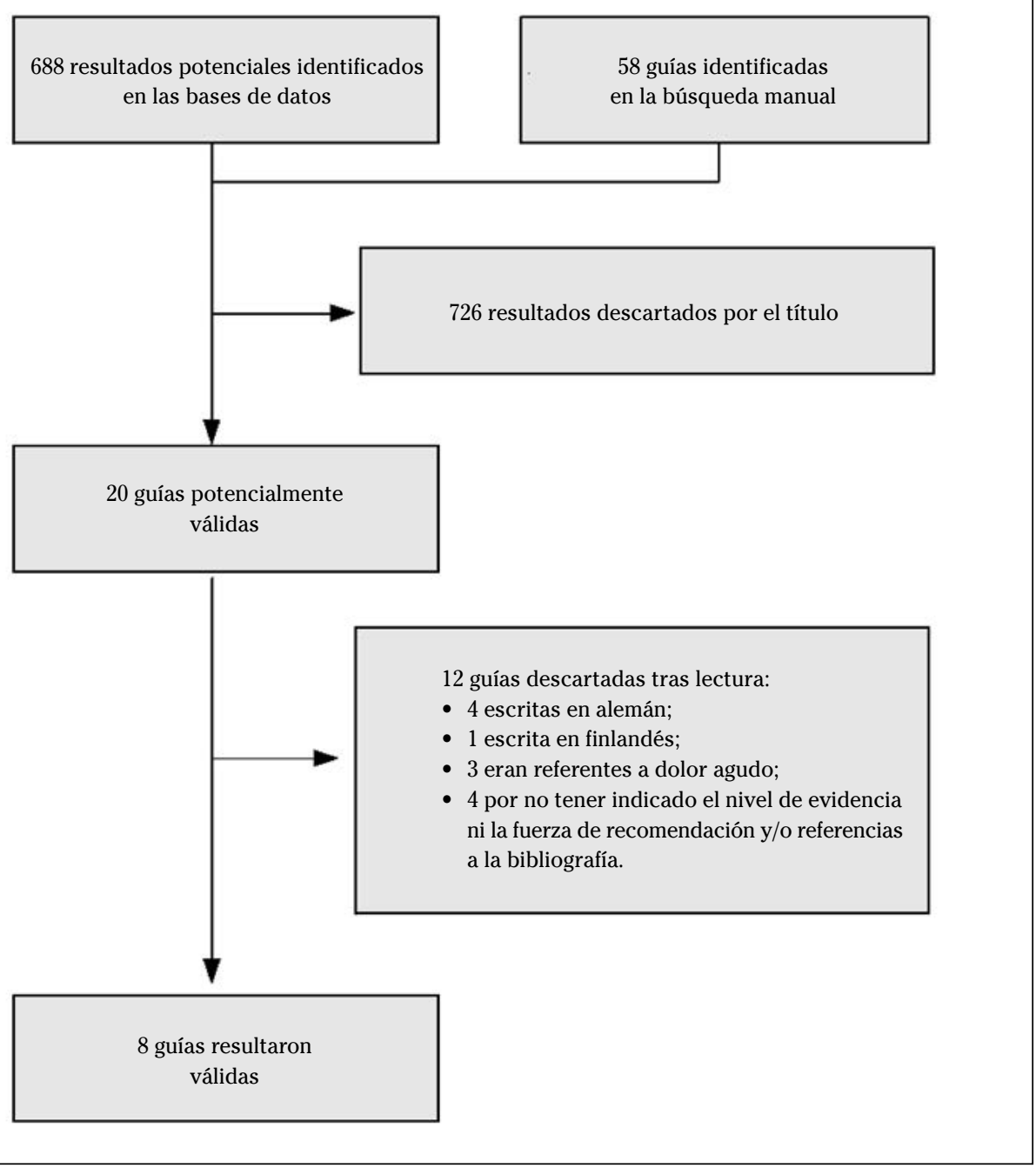

Figura 1. Flowchart de la búsqueda bibliográfica.

Existen otras opciones terapéuticas como la infiltración epidural de corticoides en caso de radiculopatía; los relajantes musculares en pacientes con espasmos musculares; y los bloqueos intrarticulares y los bloqueos o neurotomía de la rama medial para pacientes que presentan las articulaciones facetarias como origen claro de su dolor. Las guías no están de acuerdo en la aplicación de estas terapias, por lo que parece que deben de ser utilizadas como opciones terapéuticas siempre y cuando se hayan agotado las demás vías de actuación.

\section{Recomendaciones con bajo nivel de evidencia de aparición en una sola guía}

Existen tratamientos con bajo nivel de evidencia (C2 o D) que aparecen en una sola guía y no han sido incluidos en la ta- 


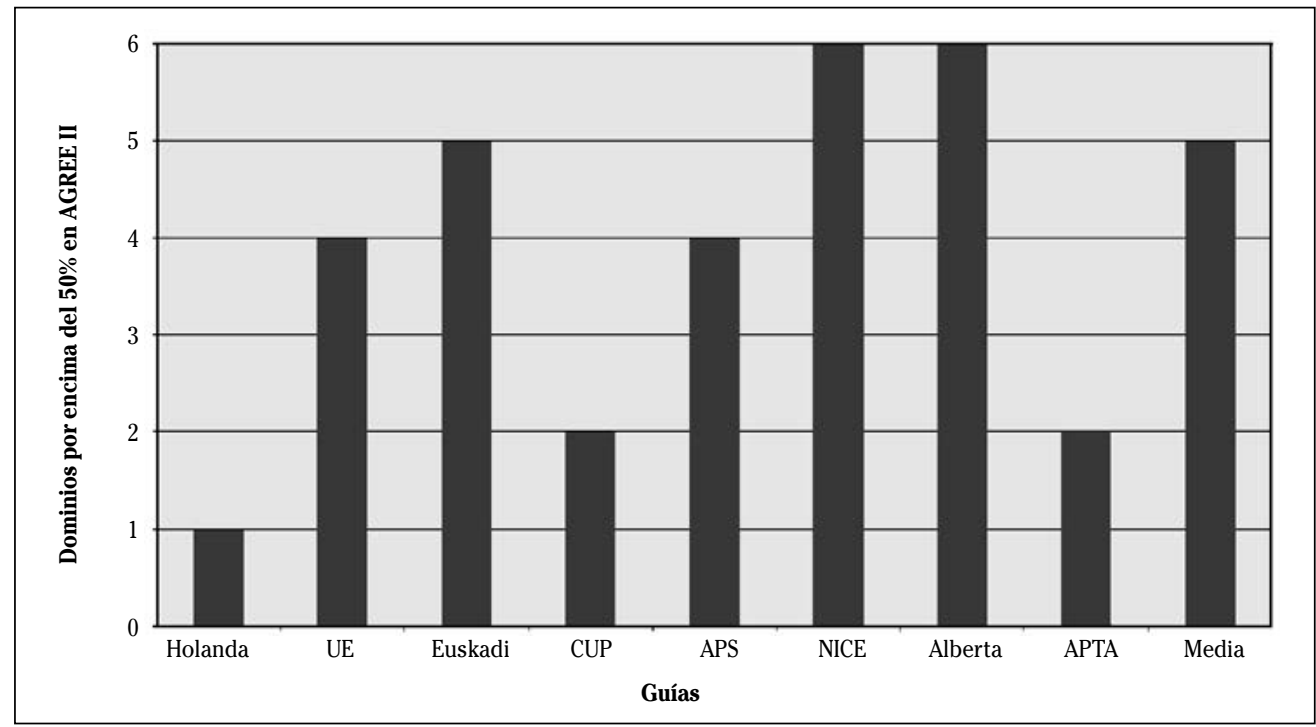

Figura 2. Número de dominios por encima del 50\% en AGREE II para cada guía.

bla 2. Estos incluyen el uso de toxina botulínica, modificación de factores de riesgo (obesidad/tabaco), balneoterapia, aspirina, termocoagulación intradiscal, terapia electrotérmica intradiscal, estimulación de la médula espinal, estimulación intramuscular, buprenorfina transdérmica y AINE tópicos.

\section{DISCUSIÓN}

El consumo de recursos desorbitado de las patologías crónicas en el Sistema Nacional de Salud justifica ampliamente las iniciativas institucionales y gubernamentales que se están llevando a cabo en España ${ }^{30,31}$. Claramente se necesita un enfoque desde la gestión de calidad para fomentar, apoyar y potenciar la implantación de modelos como éstos, aunque todavía no se conozcan bien cuáles son los factores estructurales facilitadores ${ }^{32}$. Proveer a los propios profesionales de herramientas basadas en la evidencia para iniciativas internas de mejora de la calidad, podría complementar las estrategias institucionales. Nuestros resultados muestran una variabilidad importante en el contenido de las GPC actuales en dolor lumbar crónico, por lo que ha sido necesario identificar y sintetizar abordajes terapéuticos fiables y efectivos. También se ha identificado cierta variabilidad en el método de desarrollo de las guías, sobre todo con respecto a su aplicabilidad e independencia editorial según AGREE II, que puede ser o haber sido origen de barreras al uso y adherencia de estas buenas prácticas. Por lo tanto, este trabajo pone a disposición de los profesionales la información necesaria para una gestión y práctica clínica de calidad.

El primer punto de controversia encontrado es tratar de definir el DLC. Esto se refleja en las GPC, pues encontramos que entre las seleccionadas en nuestro estudio cinco hacen referencia a DLC, en general, y tres hacen referencia a DLC inespecífico. En ocasiones se usa inespecífico como sinónimo de crónico, y aunque su significado no es el mismo, en la práctica puede ser considerado así, pues los dolores lumbares que tienden a cronificarse son aquellos en los que no existe un claro desencadenante del dolor susceptible de ser tratado.

También existen excepciones en las guías; este es el caso de recomendaciones 
dirigidas a grupos de pacientes con un origen claro del dolor, como serían los problemas de origen facetario o radiculopatías. Aunque en ocasiones se presente un dolor inespecífico sin causa clara, es necesario enfocar nuestros esfuerzos a la identificación de su origen, ya que intentar tratar el DLC como un ente único es tan inapropiado como tratar todo dolor crónico como una sola patología.

La guía de APTA $2012^{29}$ ofrece tratamientos de terapia manual para distintos grupos de pacientes dentro del DLC. Esta clasificación debe ser un requisito para el desarrollo de futuras guías de práctica clínica, pues la identificación de las terapias más efectivas para los distintos subgrupos de pacientes permitirá aumentar la efectividad de los tratamientos.

En España se ha identificado un uso inadecuado de la historia clínica, la exploración física y las radiografías durante el diagnóstico ${ }^{33}$, incluso un estudio señala que no se interrogó al paciente por señales de alarma en el 50\% de los casos de lumbalgia $^{34}$. La identificación en las guías de los subgrupos específicos de pacientes, unido a un esfuerzo de los profesionales por ser más analíticos en la búsqueda de las causas del dolor, parece que se presentará como uno de los próximos retos en el tratamiento del DLC.

$\mathrm{Si}$ atendemos a la variabilidad de los resultados de AGREE II pueden orientar sobre ciertos defectos metodológicos de las GPC, es preciso hacer un análisis más pormenorizado para poder ver la calidad del contenido para un uso práctico. De hecho, una de las limitaciones de la herramienta AGREE II es que no es útil para valorar si las recomendaciones son apropiadas y válidas ${ }^{35,36}$. Este trabajo evidencia el déficit existente en las guías en cuanto a la exhaustividad de las revisiones que se realizan para localizar la evidencia, ya que como se muestra en la tabla 2 , numerosos tratamientos (20) han sido analizados en menos de 4 guías. Es necesario mejorar por tanto la metodología de la construcción de guías de práctica clínica, para lo que se pueden usar herramientas como los manuales propuestos por $\mathrm{NICE}^{37}, \mathrm{SIGN}^{20} \mathrm{O}$
GuiaSalud $^{38}$, que permitan homogeneizar el desarrollo metodológico de las GPC.

$\mathrm{Al}$ realizar el análisis de los niveles de evidencia y fuerza de recomendación de las GPC, se muestran de nuevo bastantes inconsistencias, ya no entre las guías, si no dentro de las mismas. Una incongruencia importante es que las guías, cuando no encuentran evidencia para alguna terapia o la evidencia es muy pobre, hacen una recomendación en contra del uso de esa terapia.

Es difícil identificar la causa de estas inconsistencias, ya que como se ha dicho puede deberse a la interpretación de la evidencia por parte de los autores, pero también a que con el tiempo haya surgido nueva evidencia que clarifique el balance de beneficios, a la exhaustividad de la revisión sistemática y a la variabilidad de los sistemas de gradación de la evidencia usados en las diferentes guías.

Las recomendaciones terapéuticas de elección de las GPC analizadas no han sufrido cambios sustanciales a lo largo de los últimos años aunque la práctica clínica está lejos de seguir estas recomendaciones ${ }^{34,39}$, incurriendo en el abuso de fármacos y técnicas que no tienen una utilidad demostrada en el DLC. Es necesario por tanto fomentar el uso de GPC (o protocolos basados en evidencia) realizados de manera estandarizada y adecuada, dentro del sistema sanitario, con el objetivo de tener una fuente de información fiable de la que partan las decisiones clínicas. De esta forma, se evita no sólo un exceso de costes económicos y sociales, sino complicaciones que pueden derivar del abuso y/o adicción a fármacos, así como cirugías y pruebas diagnósticas innecesarias.

Un conjunto de indicadores publicado recientemente ${ }^{40}$ ha expuesto el bajo cumplimiento de recomendaciones similares a las expuestas en esta revisión en el tratamiento del DLC. De cinco indicadores presentados, solo uno tiene un cumplimiento aceptable, el referente al tratamiento farmacológico; los indicadores que hacen referencia a educación, ejercicio y terapia cognitivo-comportamental tienen un cumplimiento (IC 95\% exacto binomial) 
de $0 \%(0-21,8 \%), 6,67 \%(0,17-37,95 \%)$ y $0 \%$ (0-21,8\%) respectivamente, cuando estas recomendaciones son prioritarias según los resultados de esta revisión. Indicadores como los citados pueden ser una herramienta que ayude a monitorizar y mejorar la adherencia a las buenas prácticas en el tratamiento del DLC.

Las dos recomendaciones más efectivas para tratar el DLC, presentan barreras en su traslado a la práctica. En el ejercicio debemos implicar al paciente en su recuperación de forma activa. Utilizar estrategias para mantener la adherencia en caso de que el ejercicio se realice en casa, y tener en cuenta las preferencias del paciente en cuanto al tipo de ejercicio, es necesario para obtener mejores resultados ${ }^{41}$.Es recomendable que este ejercicio sea realizado bajo supervisión de un fisioterapeuta, debido al amplio rango de intervenciones disponibles (fuerza, resistencia, coordinación, etc.), por lo que seleccionar la indicada para cada paciente es esencial para el éxito del tratamiento. Otras barreras sociales o ambientales que el paciente perciba de forma individual también deben ser tenidas en cuenta a la hora de programar el ejercicio ${ }^{42}$.

La escuela de espalda incluye explicaciones de las posibles causas del dolor lumbar, anatomía y mecanismos del dolor. Se realizan ejercicios de diversa índole, dependiendo de las necesidades de los pacientes, y también incluyen consejos sobre higiene postural. Estos tres pilares forman la base de los programas de la escuela, pero no está claramente definido su contenido, la forma de aplicarlo y en qué medi$\mathrm{da}^{43}$ hacerlo. En una reciente revisión ${ }^{44}$ se han mostrado las diferencias en las herramientas de educación (consejos, vídeos, folletos, etc.) en pacientes con dolor lumbar. En el caso del dolor crónico, recomiendan información que disminuya el miedo y la ansiedad, y fomente el autocuidado, ya que mejora el pronóstico de la enfermedad.

Otras terapias como la acupuntura, el masaje, la manipulación y la movilización no están recomendadas en algunas guías, y no están reguladas completamente en España, por lo que su aplicación se puede realizar por personas con muy diferentes cualificaciones, y esto limita el éxito del tratamiento lo suficiente para no recomendar su uso generalizado ${ }^{45}$.

El tratamiento farmacológico está muy limitado temporalmente; sus beneficios no son superiores a tratamientos no farmacológicos (ejercicio y escuela de espalda), y en cambio, sus efectos secundarios son mayores. El uso continuado de AINE y/o paracetamol puede dar lugar a problemas gastrointestinales o toxicidad renal, por lo que deben usarse con moderación, de forma controlada y por cortos periodos

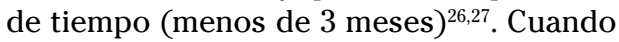
estos tratamientos fallan se puede recurrir a antidepresivos tricíclicos (a baja dosis), pero no debe mantenerse durante largos periodos. Las opciones más recomendables para el uso de la farmacoterapia son los episodios de agudización o agravamiento del dolor, y como coadyuvante a las terapias en el inicio del control del dolor, retirándose tan pronto como se consoliden los efectos de las terapias no farmacológicas de elección.

Los opioides están considerados como el último recurso farmacológico en el tratamiento del DLC y solo en caso de que todos los demás tratamientos hayan fracasado, dado los importantes efectos secundarios que presenta $a^{22,23,25-28}$. Actualmente, en Estados Unidos el uso de opioides prescritos para el tratamiento de dolores crónicos no oncológicos supone un verdadero problema de salud ${ }^{46,47}$, excediendo las 15.000 muertes al año. El inconveniente del uso de los opioides es que los estudios realizados no duran más de tres meses ${ }^{26-28,48}$, lo que limita de forma importante su aplicación clínica. El uso de opioides requiere planificar estrategias de titulación, destete y control, pactadas con el paciente a fin de evitar, en la medida de lo posible, los efectos secundarios y ofrecer una asistencia respetuosa con sus necesidades y expectativas.

Es necesario indicar que este trabajo está enmarcado dentro de un proyecto mayor ${ }^{40}$ en el que se desarrolló el sistema de gradación de la evidencia. La principal razón de usarlo frente a otros sistemas más expandidos resulta de la falta de unanimidad de éstos y las diferentes limitacio- 
nes que presentaban. En el caso de GRA$\mathrm{DE}$, por ejemplo, consume mucho tiempo y recursos, reduciendo la viabilidad de la evaluación del contenido de múltiples GPC.

En resumen, en este trabajo se han expuesto los tratamientos de forma estructurada, identificando la evidencia que los soporta, en una serie de recomendaciones que cumplen el objetivo de definir las buenas prácticas en el manejo del DLC. Las guías de práctica clínica analizadas en esta revisión ofrecen resultados similares sobre los principales tratamientos para el DLC. El ejercicio y la escuela de espalda establecen la base del tratamiento no farmacológico aportando la evidencia más sólida, además de menor incidencia de efectos secundarios. El tratamiento farmacológico se perfila como un recurso para controlar el dolor durante un corto periodo de tiempo, debiendo planificar estrategias que controlen sus efectos a largo plazo.

\section{BIBLIOGRAFÍA}

1. Chou R. Low back pain (chronic). Clin Evid [Revista Electrónica]. 2010 Jan; 2010: 1116. [Consultado 29-01-2015]: Disponible en http://www.ncbi.nlm.nih.gov/pmc/articles/ PMC3217809/

2. Kent PM, Keating JL. The epidemiology of low back pain in primary care. Chiropr Osteopat 2005; 13: 1-7.

3. Dagenais S, Caro J, Haldeman S. A systematic review of low back pain cost of illness studies in the United States and internationally. Spine J 2008; 8: 8-20.

4. Pengel LHM, Herbert RD, Maher CG, Refshauge KM. Acute low back pain: systematic review of its prognosis. BMJ 2003; 327: 1-5.

5. HaYden JA, Dunn KM, van der WindT DA, SHAW WS. What is the prognosis of back pain? Best Pract Res Clin Rheumatol 2010; 24: 167-179.

6. Henschke N, Maher CG, Refshauge KM, Herbert RD, Cumming RG, Bleasel J et al. Prognosis in patients with recent onset low back pain in Australian primary care: inception cohort study. BMJ 2008; 337: a171-a171.

7. Hooten WM, Timming R, Belgrade M, Gaul J, Goertz M, HaAKe B et al. Assessment and management of chronic pain. Bloomington: Institute for Clinical Systems Improvement, 2011.
8. Koes BW, van Tulder MW, Thomas S. Diagnosis and treatment of low back pain. BMJ 2006; 332: 1430-1434.

9. Van Tulder MW, Koes BW, Bouter LM. A costof-illness study of back pain in The Netherlands. Pain 1995; 62: 233-240.

10. Luo X, Pietrobon R, Sun SX, Liu GG, Hey L. Estimates and patterns of direct health care expenditures among individuals with back pain in the United States. Spine (Phila Pa 1976) 2004; 29: 79-86.

11. Ivanova Ji, Birnbaum HG, Schiller M, Kantor E, Johnstone BM, SwindLE RW. Real-world practice patterns, health-care utilization, and costs in patients with low back pain: the long road to guideline-concordant care. Spine J 2011; 11: 622-632.

12. Deyo RA, Mirza SK, Martin BI. Back pain prevalence and visit rates: estimates from U.S. national surveys, 2002. Spine (Phila Pa 1976) 2006; 31: 2724-2727.

13. Expósito J. Algunas claves de supervivencia para gerentes de hospitales públicos [Editorial]. Gestión Clínica y Sanitaria 2010; 12 : 119-121.

14. Pillastrini P, Gardenghi I, Bonetti F, Capra F, GucCione A, Mugnai R et al. An updated overview of clinical guidelines for chronic low back pain management in primary care. Joint Bone Spine 2012; 79: 176-185.

15. Koes BW, van Tulder M, Lin C-WC, Macedo LG, McAuley J, Maher C. An updated overview of clinical guidelines for the management of non-specific low back pain in primary care. Eur Spine J 2010; 19: 2075-2094.

16. Institute of Medicine. Clinical Practice Guidelines We Can Trust. National Academy of Sciences, editor. Washington, DC: The National Academies Press; 2011.

17. Consorcio AGREE. El Instrumento AGREE II Versión electrónica. Guiasalud. 2009. [Consultado 29-01-2015]: Disponible en http:// www.guiasalud.es/contenidos/documentos/ Guias_Practica_Clinica/Spanish-AGREE-II. pdf

18. Agency for Healthcare Research and Quality. U.S. Preventive Services Task Force (USPSTF): An Introduction (Internet). Agency for Healthcare Research and Quality, Rockville, MD. [Consultado 02-11-2014]: Disponible en http://www.ahrq.gov/professionals/ clinicians-providers/guidelines-recommendations/uspstf/index.html

19. Guyatt GH, Oxman AD, Vist GE, Kunz R, FalcKYtter Y, Alonso-Coello P et al. GRADE: An emerging consensus on rating quality of 
evidence and strength of recommendations. Chinese J Evidence-Based Med 2009; 9: 8-11.

20. Scottish Intercollegiate Guidelines Network. SIGN 50. A guideline developer's handbook. Scottish Intercollegiate Guidelines Network, editor. Edinburgo; 2011.

21. Bekkering G, Hendriks H, Koes B, Oostendorp R, Ostelo R, Thomassen J et al. Dutch physiotherapy guidelines for low back pain. Physiotherapy $2003 ; 89: 82-96$.

22. Airaksinen O, Brox JI, Cedraschi C, Hildebrandt J, Klaber-Moffett J, Kovacs F, et al. Chapter 4. European guidelines for the management of chronic nonspecific low back pain. Eur Spine J 2006; 15: S192-S300.

23. Rossignol M, Poitras S, Dionne C, Tousignant M, Truchon M, Arsenault B et al. An interdisciplinary guideline development process: the Clinic on low-back pain in Interdisciplinary Practice (CLIP) low-back pain guidelines. Implement Sci 2007; 2: 36.

24. Chou R, Huffman LH. Nonpharmacologic therapies for acute and chronic low back pain: $A$ review of the evidence for an American Pain Society/American College of Physicians clinical practice guideline. Ann Intern Med 2007; 147: 492-504.

25. Chou R, Huffman LH. Medications for acute and chronic low back pain: A review of the evidence for an American Pain Society/American College of Physicians clinical practice guideline. Ann Intern Med 2007; 147: 505-514.

26. Pérez-Irazusta I, Alcorta-Michelena I, AguirreLejarcegui G, Aristegi Racero G, Caso Martinez J, Esquisabel Martinez R et al. Guía de Práctica Clínica sobre Lumbalgia. Osakidezta y Departamento de Sanidad del Gobierno Vasco, editores. Vitoria-Gasteiz; 2007.

27. National Collaborating Centre for Primary Care (UK). Low Back Pain: Early Management of Persistent Non-specific Low Back Pain. Royal College of General Practitioners, ed. London, UK; 2009.

28. Institute of Health Economics. Guideline for the Evidence-Informed Primary Care management for Low Back Pain. 2nd edition. Alberta, Canada.; 2011.

29. Delitto A, George S, Van Dillen L, Whitman J, Sowa G, SheKelle P et al. Clinical Guidelines: Low Back Pain. J Orthop Sports Phys Ther 2012; 42: A1-A57.

30. Valdés LaRRañaga P. Evaluar el cambio organizacional hacia la cronicidad: una empresa compleja e inteligente. An Sist Sanit Navar 2014; 37: 185-188. Editorial.

31. Polanco NT, Pérez PV, Solinís RN, Solves JJM. Evaluación del nuevo enfoque en atención a la cronicidad en las organizaciones sanitarias integradas en el País Vasco. An Sist Sanit Navar 2014; 37: 189-201.

32. Coleman K, Austin BT, Brach C, Wagner EH. Evidence on the Chronic Care Model in the new millennium. Health Aff (Millwood) 2009; 28: 75-85.

33. González-Urzelai V, Palacio-Elua L, López-DeMunAIN J. Routine primary care management of acute low back pain: adherence to clinical guidelines. Eur Spine J 2003; 12: 589-594.

34. Muntión-Alfaro MT, Benítez-Camps M, BordasJulve JM, DE GisPert-Uriach B, Zamora-SÁncheZ V, Galindo-Parres C. Lumbalgia: ¿seguimos las recomendaciones de las guías? Aten Primaria 2006; 37: 215-220.

35. Knai C, Brusamento S, Legido-Quigley H, Saliba V, Panteli D, Turk E et al. Systematic review of the methodological quality of clinical guideline development for the management of chronic disease in Europe. Health Policy 2012; 107: 157-167.

36. BuRLS A. AGREE II-improving the quality of clinical care. Lancet 2010; 376: 1128-1129.

37. National Institute for Health and Clinical Excellence. The guidelines manual. National Institute for Health and Clinical Excellence, editor. London; 2012.

38. Grupo de trabajo sobre GPC. Elaboración de Guías de Práctica Clínica en el Sistema Nacional de Salud. Manual Metodológico. Instituto Aragonés de Ciencias de la Salud-I+CS, editor. Madrid: Plan Nacional para el SNS del MSC. Guías de Práctica Clínica en el SNS: I+CS No 2006/0I.; 2007.

39. Mafi JN, McCarthy EP, Davis RB, Landon BE. Worsening trends in the management and treatment of back pain. JAMA Intern Med 2013; 173: 1573-1581.

40. Saturno PJ, López Soriano F, Ángel García D, MarTínez Nicolas I. Indicadores de buenas prácticas sobre el manejo del dolor. Universidad de Murcia, editor. Murcia, España: FUINSA, Universidad de Murcia; 2013.

41. Van Middelkoop M, Rubinstein SM, Verhagen AP, Ostelo RW, Koes BW, van Tulder MW. Exercise therapy for chronic nonspecific low-back pain. Best Pract Res Clin Rheumatol 2010; 24: 193-204.

42. Slade SC, Patel S, Underwood M, Keating JL. What are patient beliefs and perceptions about exercise for nonspecific chronic low back pain? A systematic review of qualitative studies. Clin J Pain 2014; 30: 995-1005.

43. Linton SJ, Kamwendo K. Low back schools. A critical review. Phys Ther 1987; 67: 13751383. 
44. Dupeyron A, Ribinik P, Gélis A, Genty M, Claus D, HérISSON $\mathrm{C}$ et al. Education in the management of low back pain. Literature review and recall of key recommendations for practice. Ann Phys Rehabil Med 2011; 54: 319-335.

45. De Andrés Rivera Á, Ausin Hervella JL, García Estepa R, González Enríquez J, López Blanco JA, Pérez Mateos C et al. Análisis de situación de las terapias naturales. Madrid: Ministerio de Sanidad, política Social e igualdad; 2011.

46. PAulozzi LJ. Prescription drug overdoses: a review. J Safety Res 2012; 43: 283-289.
47. Warner M, ChEn LH, MAKUC DM. Increase in fatal poisonings involving opioid analgesics in the United States, 1999-2006. NCHS Data Brief [Revista electrónica]. 2009; 1-8. [Consultado 05-11-2014]: Disponible en http://www.cdc. gov/nchs/data/databriefs/db22.htm

48. Martell BA, O'Connor PG, Kerns RD, Becker WC, Morales KH, Kosten TR et al. Systematic review: Opioid treatment for chronic back pain: Prevalence, efficacy, and association with addiction. Ann Intern Med 2007; 146: 116-127. 